\title{
Linking Amygdala Persistence to Real-World Emotional Experience and Psychological Well-Being
}

\author{
${ }^{\circledR}$ Nikki A. Puccetti, ${ }^{1}{ }^{\circledR}$ Stacey M. Schaefer, ${ }^{2}{ }^{\circledR}$ Carien M. van Reekum, ${ }^{3}$ Anthony D. Ong, ${ }^{4}{ }^{\circledR}$ David M. Almeida, ${ }^{5}$ \\ ${ }^{\circledR}$ Carol D. Ryff, ${ }^{6}{ }^{\circledR}$ Richard J. Davidson, ${ }^{2,6}$ and ${ }^{\circledR}$ Aaron S. Heller ${ }^{1}$ \\ ${ }^{1}$ Department of Psychology, University of Miami, Coral Gables, Florida 33124, ${ }^{2}$ Center for Healthy Minds, University of Wisconsin-Madison, \\ Madison, Wisconsin 53703, ${ }^{3}$ School of Psychology and Clinical Language Science, University of Reading, Reading RG6 6AL, United Kingdom, \\ ${ }^{4}$ Department of Human Development, Cornell University, Ithaca, New York 14853, ${ }^{5}$ Department of Human Development and Family Studies and \\ Center for Healthy Aging, The Pennsylvania State University, University Park, Pennsylvania 16802, and ${ }^{6}$ Department of Psychology, University of \\ Wisconsin-Madison, Madison, Wisconsin 53706
}

Neural dynamics in response to affective stimuli are linked to momentary emotional experiences. The amygdala, in particular, is involved in subjective emotional experience and assigning value to neutral stimuli. Because amygdala activity persistence following aversive events varies across individuals, some may evaluate subsequent neutral stimuli more negatively than others. This may lead to more frequent and long-lasting momentary emotional experiences, which may also be linked to selfevaluative measures of psychological well-being (PWB). Despite extant links between daily affect and PWB, few studies have directly explored the links between amygdala persistence, daily affective experience, and PWB. To that end, we examined data from 52 human adults (67\% female) in the Midlife in the United States study who completed measures of PWB, daily affect, and functional MRI (fMRI). During fMRI, participants viewed affective images followed by a neutral facial expression, permitting quantification of individual differences in the similarity of amygdala representations of affective stimuli and neutral facial expressions that follow. Using representational similarity analysis, neural persistence following aversive stimuli was operationalized as similarity between the amygdala activation patterns while encoding negative images and the neutral facial expressions shown afterward. Individuals demonstrating less persistent activation patterns in the left amygdala to aversive stimuli reported more positive and less negative affect in daily life. Further, daily positive affect served as an indirect link between left amygdala persistence and PWB. These results clarify important connections between individual differences in brain function, daily experiences of affect, and well-being.

Key words: amygdala; daily affect; emotion; fMRI; neural dynamics; representational similarity analysis

Significance Statement

At the intersection of affective neuroscience and psychology, researchers have aimed to understand how individual differences in the neural processing of affective events map onto to real-world emotional experiences and evaluations of well-being. Using a longitudinal dataset from 52 adults in the Midlife in the United States (MIDUS) study, we provide an integrative model of affective functioning: less amygdala persistence following negative images predicts greater positive affect (PA) in daily life, which in turn predicts greater psychological well-being (PWB) seven years later. Thus, day-to-day experiences of PA comprise a promising intermediate step that links individual differences in neural dynamics to complex judgements of PWB.

Received June 29, 2020; revised Feb. 3, 2021; accepted Feb. 24, 2021.

Author contributions: S.M.S., C.M.v.R., A.D.O., D.M.A., C.D.R., and R.J.D. designed research; S.M.S., C.M.v.R., A.D.O., D.M.A., C.D.R., and R.J.D. performed research; N.A.P. and A.S.H. analyzed data; N.A.P. and A.S.H. wrote the paper.

Publicly available data from the MIDUS study were used for this research. Since 1995, the MIDUS study has been funded by the following: John D. and Catherine T. MacArthur Foundation Research Network and National Institute on Aging Grants P01-AG020166 and U19-AG051426.

R.J.D. serves on the board of directors for the non-profit organization Healthy Minds Innovations. All other authors declare no competing financial interests.

Correspondence should be addressed to Aaron S. Heller at aheller@miami.edu.

https://doi.org/10.1523/JNEUROSCI.1637-20.2021

Copyright $\odot 2021$ the authors

\section{Introduction}

Psychological well-being (PWB) captures one's perceived self-acceptance, positive relations with others, autonomy, environmental mastery, purpose in life, and personal growth (Ryff, 1989; Ryff and Keyes, 1995). Relatively more enduring, trait-like judgements of PWB are linked to relatively more transient positive and negative emotional states in the real-world (Burns and Ma, 2015; Rush et al., 2019). Specifically, higher PWB is associated with greater positive affect (PA; Burns and Ma, 2015) and lower negative affect (NA; Rush et al., 2019) in daily life. A key question is what neurobiological processes give rise to these subjective 
affective experiences and judgements. One promising source of these individual differences may be the time course of amygdala activity. (Sander et al., 2003).

The amygdala is necessary for threat detection and threat conditioning-the assigning of value to neutral stimuli (LeDoux, 1996). The amygdala is also implicated in a range of affective processes, including general detection of salience (Davis and Whalen, 2001; Sander et al., 2003; Kober et al., 2008), facial processing (Todorov et al., 2008), and experiencing fear (LeDoux, 2000). In particular, the amygdala supports negative appraisals of putatively neutral stimuli when they are preceded by an unrelated, aversive stimulus (Lapate et al., 2016). Individual differences in amygdala response to fearful faces predict how negatively a subsequent, unrelated neutral stimulus is appraised (Lapate et al., 2016, 2017). This biasing effect may be because of persistence of amygdala activity following emotionally evocative stimuli that "spills over" into the encoding of the subsequent neutral stimulus (Tambini et al., 2017; Grupe et al., 2018).

Functional MRI (fMRI) research suggests that individual differences in the degree of amygdala activity persistence after aversive events is associated with individual differences in affective style. Schuyler et al. (2014) found that more persistent amygdala activity following negative images predicted higher levels of neuroticism. Additionally, greater amygdala persistence following negative images was linked to lower likeability ratings of subsequent neutral faces. Similarly, greater amygdala persistence following negative words has been found in patients with major depressive disorder (Siegle et al., 2002). These studies demonstrate that amygdala persistence is linked to individual differences in affective style.

However, the existing literature has relied on univariate amygdala analyses that cannot account for the multivariate representation of affective stimuli. Assessing persistence by averaging activity over the amygdala removes fine-grained, spatial activity patterns that carry detailed information about stimulus properties. In contrast, multivariate approaches, such as representational similarity analysis (RSA), preserve the voxel-wise pattern of activation and compare the neural representations of different types of stimuli. Although RSA was initially used to examine similarity in visual perception (Kriegeskorte and Bandettini, 2007; Kriegeskorte et al., 2008), it has been applied to identify similarity in affective processing (Ölander et al., 2017; Brooks and Freeman, 2018). Given that we rarely encounter a single stimulus in isolation, the dynamics of amygdala representation across temporally-related stimuli are paramount to understanding individual differences in well-being. Therefore, by capturing the commonalities in the voxel-wise pattern of amygdala activity among affective stimuli, compared with the pattern of activity among subsequent neutral facial expressions, RSA could yield a more accurate and ecologically valid metric of amygdala persistence.

To that end, the current study used RSA to test whether individual differences in amygdala persistence following affective images was linked with daily affective experience and PWB. We analyzed a Midlife in the United States (MIDUS) subsample that completed a PWB questionnaire, daily telephone interviews of affective experience, and an fMRI scan. We hypothesized that those with less amygdala persistence following negative visual stimuli, reflected in less similarity between voxel-wise neural representations of negative stimuli and neutral facial expressions that follow negative stimuli, would report higher daily PA and less daily NA. In line with previous work, we further hypothesized that higher reports of daily PA and less NA would correspond to greater PWB. Lastly, using a mediational framework, we tested whether daily affect provided a pathway by which amygdala persistence was linked with PWB.

\section{Materials and Methods}

\section{Participant characteristics}

Data were collected from 2004 to 2009 as a part of the MIDUS-II Longitudinal Study of Health and Well-being (http://www.midus.wisc. edu/), which recruited a national sample (ages 35-85) through random digit dialing. Participants first completed self-report questionnaires $(n=4963)$, a random subsample then completed a series of eight daily consecutive telephone interviews to assess daily affect $(n=2022)$, and a further subgroup able and willing to travel to the Midwest included neuroscience assessments, including an fMRI scan $(n=72)$. Two participants did not complete all five functional runs of the MRI task; eight were removed for excessive motion (sudden spikes, gradual drift, or both, that exceeded $2 \mathrm{~mm}$ ). Nine of the remaining participants did not complete the daily diary study. Finally, one participant was excluded from the analysis for reporting levels of daily NA greater than 3 SDs from the group mean. This resulted in 52 participants that completed MRI, daily diary, and self-reported PWB for the analyses [39-76years old $(\mathrm{M}=57.74, \mathrm{SD}=10.5), 67 \%$ female, $69 \%$ white, $29 \%$ African American, $2 \%$ Native American or Alaskan Native]. There were five sets of twins in the sample. Lastly, 31 of these subjects repeated the self-report questionnaires pertaining to $\mathrm{PWB}$ again seven years later $(\mathrm{SD}=1.6$, range $=5$ 9 years).

On average, the first measurement of PWB was completed $729 \mathrm{~d}$, or 24.3 months, before the daily diary procedure $(S D=430 \mathrm{~d}$, range $=136-$ $1533 \mathrm{~d}$ ) and $1230 \mathrm{~d}$, or 41 months, before the fMRI scan $(\mathrm{SD}=372 \mathrm{~d}$, range $=348-1669 \mathrm{~d}$ ). Thirty-one participants completed the daily diaries before the fMRI session by an average of $999 \mathrm{~d}(\mathrm{SD}=347 \mathrm{~d}$, range $=408-$ $1491 \mathrm{~d}$ ). The other 21 participants completed the fMRI scan before the daily diary procedure by an average of $231 \mathrm{~d}(\mathrm{SD}=59$, range $=161-$ $386 \mathrm{~d})$. Because significant time passed between each measurement and time lag varied by participants, each relevant time lag is included as a covariate in the analysis. Therefore, significant relationships from the analyses are likely to reflect stable, meaningful connections that withstand long and variable measurement gaps. Data and documentation for MIDUS 1, MIDUS 2, MIDUS 3, and all MIDUS projects are available to the public for downloading at the Inter-university Consortium for Political and Social Research (ICPSR) website (https://www.icpsr.umich. edu/web/ICPSR/series/203).

\section{Experimental design and statistical analyses}

$P W B$

Participants completed a 42-item measure of PWB (Ryff, 1989; Ryff and Keyes, 1995). This measure contains subscales for six domains of PWB, including self-acceptance, positive relations with others, autonomy, environmental mastery, purpose in life, and personal growth. Each subscale is comprised of seven items that are rated on a 1-7 scale ("strongly agree" to "strongly disagree"). Items that indicate greater PWB are reverse coded such that higher overall scores reflect higher PWB. The total score for the measure is calculated by averaging the score from each of the subscales. This measure of PWB has been shown to be reliable over the span of six weeks (test-retest reliability for the six scales $>0.8$; Ryff, 1989) and stable over a 9- to 10-year period (Christensen and Mendoza, 1986; Heller et al., 2013; Radler et al., 2018). The subset of 31 subjects that completed this measure again seven years later show an intraclass correlation of 0.797 between their two PWB scores. Thus, PWB scores likely reflect stable individual differences in well-being that can be linked with daily diary and fMRI data collected months or years later.

\section{Daily diary procedure}

The daily diary protocol included telephone interviews each evening for eight consecutive evenings. Participants reported on stressful events and a range of positive and negative emotions since the time they woke up. The frequency with which participants experienced each emotion that day was rated on a five-point scale $(0=$ none of the time, $4=$ all of the 
A fMRI Task Design $\quad$ B Quantifying Amygdala Persistence C Daily Affective Experiences

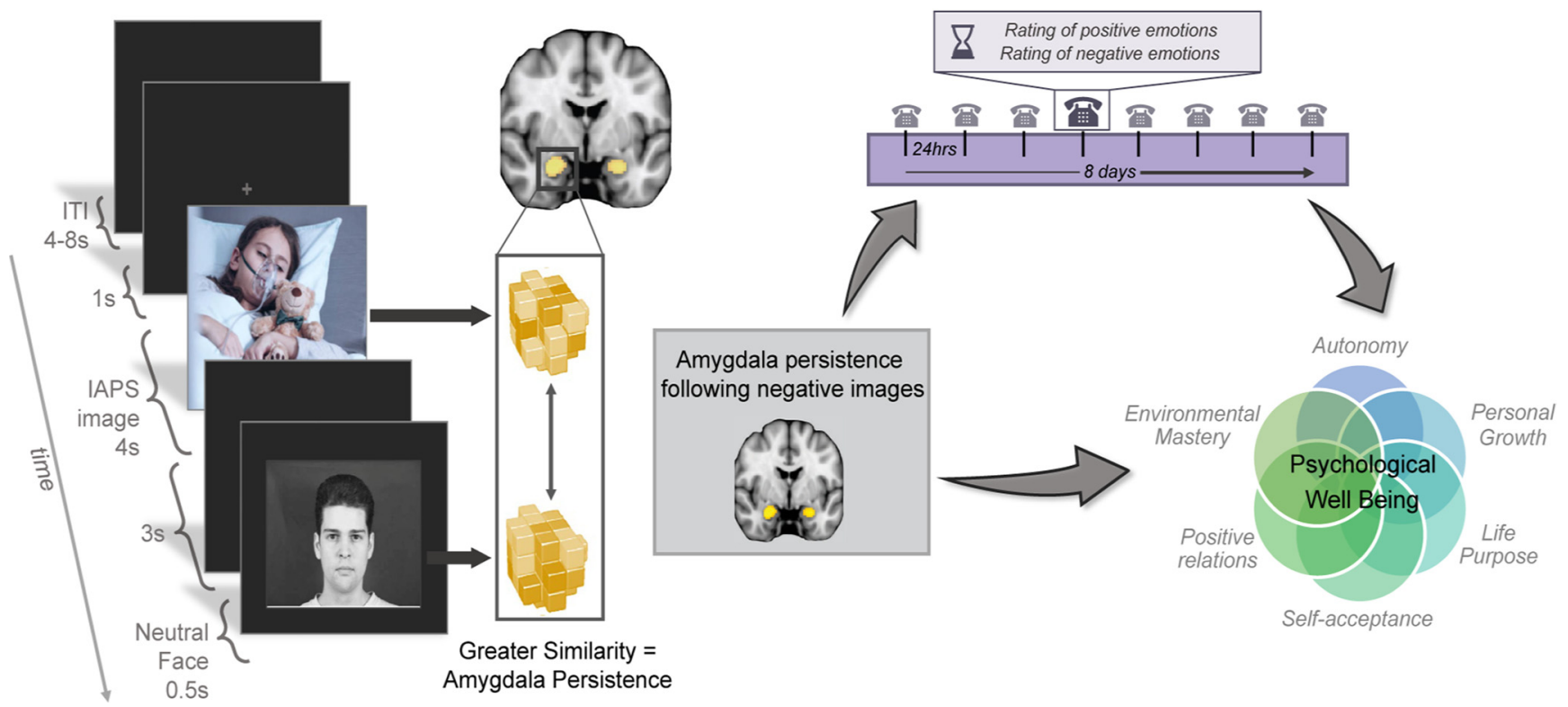

Figure 1. Study design schematic. $A$, fMRI task design. Participants viewed 60 negative, neutral, and positive IAPS images for $4 \mathrm{~s}$. When viewing the image, participants indicated whether the image was negative, neutral, or positive via a button press. Images were followed by either a neutral facial expression displayed for $0.5 \mathrm{~s}$ or a black screen. On neutral facial expression trials, the face was displayed $3 \mathrm{~s}$ after the offset of the IAPS image. We note that there were 20 trials with faces displayed $1 \mathrm{~s}$ after the image, which are not pictured here, that were not analyzed in relation to the affective outcomes of interest. Images here are examples of images seen not actual images used. $\boldsymbol{B}$, Quantifying amygdala persistence. An RSA was conducted to calculate the voxel-wise pattern similarity in the amygdala between the negative images and the neutral faces that followed them. $\boldsymbol{C}$, Daily affective experience. Participants reported on the degree to which they experienced 13 positive and 14 negative emotions throughout their day. This information was collected over the telephone to an interviewer for eight consecutive days. Individual differences in daily affective experience were used as a mediator linking the metric of amygdala persistence with PWB. PWB, psychological well-being; RSA, representational similarity analysis.

time). Thirteen PA items were rated: "in good spirits," "cheerful," "extremely happy," "calm and peaceful," "satisfied," "full of life," "close to others," "like you belong," "enthusiastic," "attentive," "proud," "active," and "confident"; and fourteen NA items were rated: "restless or fidgety," "nervous," "worthless," "so sad nothing cheered you up," "everything required effort," "hopeless," "lonely," "afraid," "jittery," "irritable," "ashamed," "upset," "frustrated," and "angry." Cronbach's $\alpha$ for the PA scale $=0.96$ and for the NA scale $=0.91$, based on reliability calculations recommended by Raudenbush and colleagues (Raudenbush et al., 1991; Charles et al., 2016, 2019). These items were averaged to create a daily PA and NA score. The average across all of a participant's daily interviews was taken to reflect their mean PA and NA.

\section{fMRI procedure and acquisition}

Participants completed an image-viewing task in which they saw 60 positive, 60 negative, and 60 neutral images from the International Affective Picture System (IAPS; Fig. 1; Lang et al., 2008). These images were matched for luminosity, picture complexity (using the jpeg file size as an index of complexity) and social content (van Reekum et al., 2018), and the positive and negative picture sets were equally arousing on average. Following a $1 \mathrm{~s}$ fixation screen, each image was presented for $4 \mathrm{~s}$, and participants indicated with a manual response whether the image was positive, negative or neutral in valence. Following IAPS slides was either a neutral facial expression (1 or $3 \mathrm{~s}$ following the IAPS image offset), or a black screen with a fixation cross but no face. Faces were one of 30 male faces from the XM2VTSDB multimodal face database (Messer et al., 1999) presented for $0.5 \mathrm{~s}$ (Fig. 1). Participants were asked to simply view the faces and made no response to these stimuli. The total intertrial interval (including the face presentations for trials where faces followed an IAPS picture) was selected from an exponential distribution and varied from 5.5-17.6 $\mathrm{s}$ with an average duration $=8.89 \mathrm{~s}$, and consisted of a black screen with a white fixation cross.

A standard clinical whole-head quadrature head coil was used. Five functional runs were collected. Functional images were obtained using a T2*-weighted, echoplanar images [EPIs; 30 sagittal slices, 4-mm thickness with 1 -mm gap; $3.75 \times 3.75 \mathrm{~mm}$ in-plane $(64 \times 64$ voxels); FOV $=240$; repetition time (TR)/echo time (TE)/flip, $2000 \mathrm{~ms} / 30 \mathrm{~ms} /$ $60^{\circ} ; 262$ whole-brain volumes per run]. Additionally, a high-resolution T1-weighted anatomic image was obtained (T1-weighted inversion recovery fast gradient echo; $256 \times 256$ in-plane resolution; $240-\mathrm{mm}$ FOV; $124 \times 1.1-\mathrm{mm}$ axial slices).

\section{fMRI analysis}

fMRI data were resampled to $2 \times 2 \times 2 \mathrm{~mm}$, then preprocessed and analyzed using AFNI (Cox, 1996), ANTs (Avants et al., 2011), and FSL (Jenkinson et al., 2012). T1 images were skull-stripped using FSL's BET function. For functional data, the first four volumes of each run were discarded before analysis and then functional images were despiked using the $3 \mathrm{dDespike}$ program from the AFNI toolbox. Motion correction was performed with the ANTs toolbox; functional images were first aligned within each run to the mean image, then all runs were aligned to the mean image from the first functional run. Next, using ANTs, nonlinear spatial normalization was applied to the functional data to match the MNI152 template. The normalized functional images were then brainmasked and scaled using FSL.

The preprocessed data were input to AFNI's 3dDeconvolve to test a general linear model (GLM) with task and nuisance regressors. The 5 functional runs were concatenated, and the model was estimated using these concatenated data. There were nine task regressors: three regressors for the IAPs (one for each of the positive, negative, neutral conditions; convolved for $4 \mathrm{~s}$ ), three regressors for neutral facial expressions $1 \mathrm{~s}$ after an image (one for each of the positive, negative, neutral images it follows; convolved for $0.5 \mathrm{~s}$ ), and three for neutral facial expressions $3 \mathrm{~s}$ later (again one for each of the positive, negative, neutral images it follows; convolved for $0.5 \mathrm{~s}$ ). The six standard motion regressors were included, as well as their derivatives, and the square of each of those 12 parameters, resulting in 24 motion parameters in the model to remove sources of variance because of motion (Satterthwaite et al., 2013). At the individual level, this GLM yielded wholebrain maps of $\beta$ coefficients for each voxel for each task condition.

\section{RSA}

We used RSA to measure individual differences in the persistence of amygdala activity patterns in response to different classes of stimuli: 
Table 1. Means, SDs, and Pearson bivariate correlation coefficients between affective variables and amygdala persistence

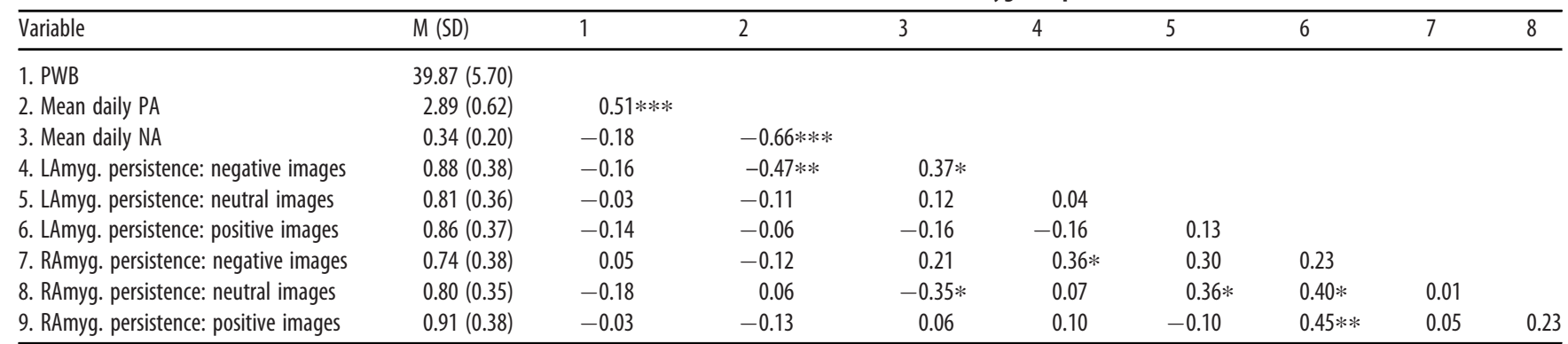

$N=52$. Mean daily NA is square root transformed here. Correlations are FDR-corrected. PWB, psychological well-being; NA, negative affect; $\mathrm{PA}$, positive affect; Amyg., amygdala; $\mathrm{L}$, left; $\mathrm{R}$, right; $* p<0.05, * * p<0.01$, $* * * p<0.001$.

negative images and neutral facial expression that follow negative images. In essence, this analysis quantifies the how closely the representation of neutral face expressions that are presented after negative stimuli resemble the representation of actual negative images in the amygdala. To accomplish this, the subject-level, whole-brain $\beta$ maps from the GLM were inputted to the RSA MATLAB toolbox (Nili et al., 2014). $\beta$ maps were masked to isolate voxels of the left and right amygdala thresholded at 50\% using the Harvard Oxford Subcortical Brain Atlas (Desikan et al., 2006). Given evidence from fMRI studies for amygdala laterality in some processing of affective stimuli (Baas et al., 2004; Dyck et al., 2011; Murphy et al., 2020), we examined the left and right amygdala separately. The $2 \times 2 \times 2$-mm left amygdala mask contained 240 voxels and the right amygdala mask contained 280 voxels. These masks correspond to $\sim 27$ and 32 voxels, respectively, in the native sampling space. The persistence of left and right amygdala activity from affective images to subsequent neutral facial expressions was quantified in the following way: we extracted the pattern of left and right amygdala activity in response to the IAPS image as well as in response to the neutral faces. Both patterns were reshaped into a $1 \times n$ vector and correlated with one another. These correlation values were Fisher $\mathrm{Z}$ transformed then subtracted from 1 to calculate the representational distance, or dissimilarity, between the two patterns. This typical dissimilarity metric yields values which range from 0 to 2 , with greater numbers reflecting greater dissimilarity. Because this analysis was focused on persistence (a measure of similarity rather than dissimilarity), we inverted the dissimilarity metrics from the RSA analysis. Also, we elected to use the neutral facial expressions presented at $3 \mathrm{~s}$ post-IAPS offset, rather than 1s, for our metric of amygdala persistence given the sluggish nature of the BOLD signal (Lindquist et al., 2009).

For the primary analyses, this persistence metric was calculated by including all stimuli from all runs into a single GLM. However, because within-run temporal autocorrelation could bias similarity metrics, we performed additional analyses in which each fMRI run was analyzed separately. This alternative method reduces potential biases introduced by temporal collinearity among events within the same run. Once each run was modeled with a separate GLM, this similarity metric was calculated by individually estimating the dissimilarity among all between-run, image-face comparisons (e.g., similarity of amygdala IAPS activity of run 1 with amygdala face activity in run 2 , run 3 , etc.). Once dissimilarity metrics for all 20 IAPS-face permutations were calculated, they were averaged together. Importantly, this average value excludes the within-run comparisons (e.g., there is no comparison among run 1 IAPS slides with run 1 faces). Replicating our analyses using this potentially less biased metric demonstrates that the primary effects are unlikely to be a product of biased estimates driven by within-run temporal correlation.

Associations between amygdala persistence and daily affect

After assessing zero-order correlations, we tested whether amygdala persistence following negative images was associated with mean daily PA and NA in multiple linear regressions. These regressions included covariates of age, gender, race, twin status, time between visits, and number of telephone diary interviews completed. Specifically, dummy-coded variables were included for race categories and each twin pair. We included persistence following neutral and positive images as covariates in all models to confirm that any effects were specific to amygdala persistence following negative images. We conducted an identical analysis, but with patterns of activity in the left and right occipital pole, the right and left nucleus accumbens (NAcc), and the medial prefrontal cortex (mPFC) to test whether effects were specific to the amygdala. Similar to the amygdala, these regions were taken from the Harvard Oxford Brain Atlas and thresholded at 50\% (Desikan et al., 2006). We selected the occipital pole because we did not expect persistence in these visual regions to be associated with daily affect. However, we selected the NAcc and the mPFC to explore whether other regions that are often implicated in affective processing show similar effects as the amygdala. False discovery rate (FDR; Benjamini and Hochberg, 1995) correction was applied to these correlation matrices by using the raw $p$ value vector as input to the p.adjust command in the stats package (i.e., p.adjust(uncorrected_ps, method $=$ "fdr"). p.adjust also requires an argument for the number of unique pairwise comparisons of interest. In the 9 by 9 correlation matrix shown in Table 1 , for example, the number of tests is 36 or the length of the raw $p$ value vector. Further, $p$ values obtained from multiple regression models for relationships involving variables of interest (daily NA, PA, PWB, and amygdala persistence) were concatenated into a single vector and FDR-corrected using p.adjust.

To test whether the RSA-derived persistence was superior to a univariate measure of persistence in predicting affect, we computed the difference between univariate amygdala responses to the IAPs images and the neutral facial expressions. Although it has been argued that withinperson difference scores in neural activity may be unreliable as individual difference measures (Infantolino et al., 2018), this metric was most analogous to the RSA-derived persistence metric. We calculated mean $\beta$ value across the voxels in the left and right amygdala for negative, neutral, and positive IAPs images and also for those faces that followed those images. We then took the absolute value of the difference between the IAPs images and the face stimuli for each valence condition to obtain scores analogous to similarity, or representational distance. These univariate differences scores were then examined as predictors of daily affect and PWB in correlations and multiple regression models similar to the multivariate persistence metrics.

Finally, path analyses were conducted using version $0.6-4$ of the 'lavaan' package in R (Rosseel, 2012) to test whether amygdala persistence predicted PWB, via daily affect. First, with the full sample, we assessed whether daily affect mediated the relationship between amygdala persistence and the first measurement of PWB. While this model takes advantage of all available data, the outcome precedes the predictors in time. To overcome this challenge, we also tested a similar model with the subset of participants who had completed a measurement of PWB after their fMRI and diaries and used this second measurement of PWB as the outcome. Further, while traditional mediation frameworks required that a significant total effect be present for amygdala persistence and PWB (Baron and Kenny, 1986), current methodological models of mediation suggest that this direct pathway is not necessary to detect mediation (Hayes, 2009). The significance of the path coefficients in these models was determined by using the Wald $\mathrm{z}$-statistic, which divides the 

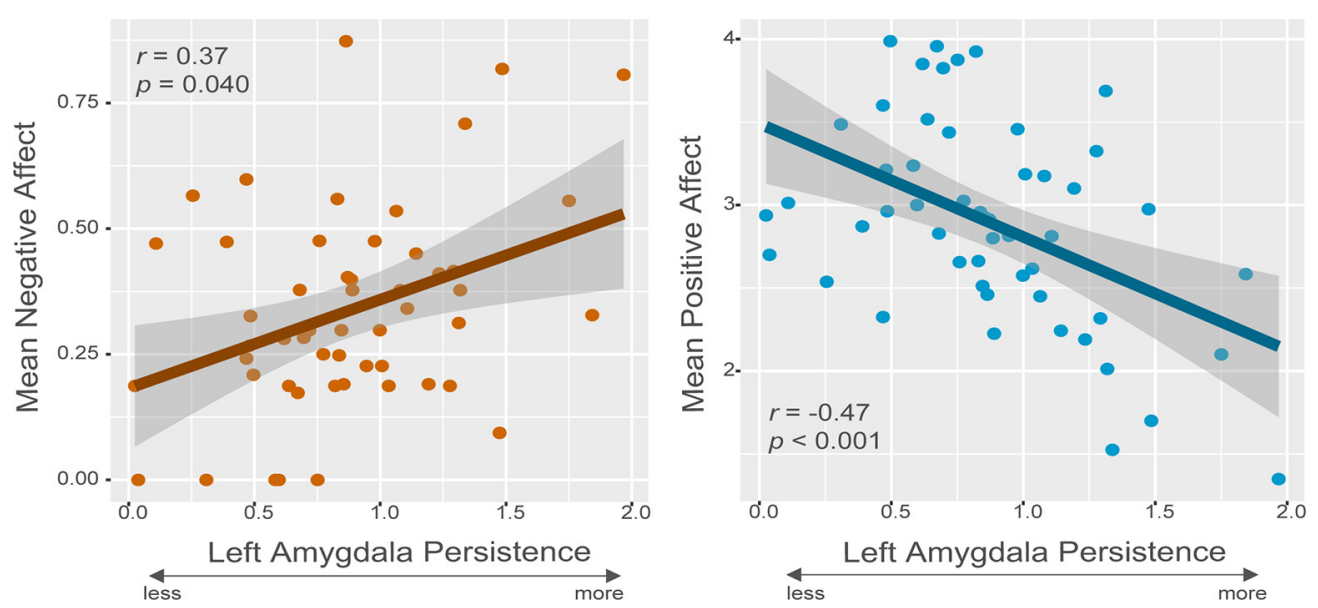

Figure 2. Left amygdala persistence following negative images is associated with daily negative and positive affect. Zero-order Pearson correlation plots between left amygdala persistence following negative images and mean negative and positive affect from the daily diaries with a gray standard error ribbon. The square root transformed mean NA is shown here. $N=52$.

parameter estimate by its standard error value. The path model also included the identical covariates as above.

\section{Results}

\section{Descriptive statistics}

The mean of the composite PWB score was 39.87 ( $\mathrm{SD}=5.70$, range $=22.17-48.50)$. For the 31 participants that reported on PWB approximately seven years later, the mean was 40.33 $(\mathrm{SD}=4.63$, range $=28.75-48.50)$. Participants completed an average of 7.5 daily telephone interviews $(S D=0.87$, range $=4-8$ ). The mean daily NA was $0.15(\mathrm{SD}=0.17$, range $=0-0.76)$, mean daily PA was 2.89 ( $S D=0.62$, range $=1.35-3.99)$. Daily affect variables were assessed for acceptable skew and kurtosis based on recommendations by Kline (i.e., skewness $<|2|$ and kurtosis $<1$ 7|; Kline, 2015) with the 'psych' $\mathrm{R}$ package, version 1.8.12 (Revelle, 2020). While mean PA demonstrated acceptable skew $(-0.23)$ and kurtosis $(-0.22)$, mean NA showed a positive skew that approached unacceptable (1.89) with acceptable kurtosis (3.34). As a result, mean NA was square root transformed (transformed skewness $=0.59$ and kurtosis $=0.25$ ). Results from all analyses remained unchanged when tested with the raw NA values. Descriptive statistics for all variables are shown in Table 1.

Left amygdala persistence following a negative image ranged from 0.03 to 1.97 , with a mean of $0.88(S D=0.43)$. Left amygdala persistence activity following a neutral image ranged from 0.06 to 1.78 , with a mean of $0.80(\mathrm{SD}=0.41)$. Left amygdala persistence following a positive image ranged from 0.01 to 1.74 , with a mean of $0.84(\mathrm{SD}=0.42)$.

Right amygdala persistence following a negative image ranged from 0.00 to $1.79(\mathrm{M}=0.71, \mathrm{SD}=0.44)$. Right amygdala persistence following a neutral image ranged from 0.10 to $2.14(\mathrm{M}=0.79$, $\mathrm{SD}=0.40$ ) and right amygdala persistence following a positive image ranged from 0.01 to $1.97(\mathrm{M}=0.89, \mathrm{SD}=0.44)$. A repeated measures ANOVA assessed differences in persistence by hemisphere (left and right) across the three image valence conditions. There was no main effect of hemisphere $\left(F_{(1,51)}=0.80, p=0.372\right)$ or valence $F_{(2,102)}=0.97, p=0.382$, nor was there an interaction between hemisphere and valence $\left.F_{(2,102)}=1.80, p=0.167\right)$.

Similarity in left and right amygdala persistence was also observed in Pearson bivariate correlations. There were positive correlations between left and right amygdala persistence following a negative image $(r=0.36, p=0.030)$, a neutral image $(r=0.36, p=0.030)$, and a positive image $(r=0.45, p=0.003)$.
Links between amygdala persistence and daily affect

Pearson bivariate correlations (Table 1) revealed that left amygdala persistence following negative images was associated with daily mean NA $(r=0.37, p=0.040$; Fig. $2 A)$ and daily mean PA $(r=-0.47, p<0.001$; Fig. $2 B)$. Left amygdala persistence following neutral images was not associated with daily mean NA $(r=0.12, p=0.626)$ or PA $(r=-0.11, p=0.645)$. Similarly, persistence following positive images was unrelated to mean NA $(r$ $=-0.16, p=0.540)$ or PA $(r=-0.06, p=0.810)$. In contrast with the left amygdala, right amygdala persistence following negative images was not significantly related to daily mean NA $(r=0.21$, $p=0.370)$ or daily mean PA $(r=-0.12, p=0.626)$. The relationship between amygdala persistence and daily NA was not significantly different between the left and right amygdala $\left(t_{(51)}=1.04\right.$, $p=0.305)$ in a test of the difference between two correlated correlations in the psych package in $\mathrm{R}$ (version 1.8.12; Steiger, 1980; Revelle, 2020). However, the relationship between amygdala persistence and daily PA was significantly stronger in the left amygdala compared with the right $\left(t_{(51)}=-2.45, p=0.018\right)$.

Given the associations between left amygdala persistence following negative images and daily affect, we tested the robustness of these effects in a multiple regression model that included relevant covariates. The effect of left amygdala persistence following the negative images on daily affect held when including covariates in multiple regressions. Greater amygdala persistence predicted more daily NA $(b=0.24$, $\left.t_{(40)}=3.46, p=0.003, B=0.50\right)$ and less daily PA $(b=-0.97$, $\left.t_{(40)}=-4.84, p<0.001, B=-0.67\right)$ when controlling for age, gender, race, twin status, the time between visits, and the number of diaries completed. The relationship between left amygdala persistence following negative images and daily NA was also specific, as persistence following neutral images $\left(b=0.07, t_{(38)}=1.00, p=0.448, B=0.014\right)$, or following positive images $\left(b=-0.03, t_{(38)}=-0.41, p=0.730, B=-0.06\right)$, did not attenuate the relationship between left amygdala persistence following negative images and daily NA $(b=0.23$, $\left.t_{(38)}=3.19, p=0.006, B=0.48\right)$ when included in the model (adjusted $R^{2}=0.17$; Table 2). Similarly, left amygdala persistence following neutral images $\left(b=-0.08, t_{(38)}=-0.40\right.$, $p=0.730, B=-0.06)$, or following positive images $(b=$ $\left.-0.11, t_{(38)}=-0.56, p=0.700, B=-0.08\right)$, did not attenuate the effect of persistence following negative images on daily PA $\left(b=-0.97, t_{(38)}=-4.69, p<0.001, B=-0.67\right.$; full model adjusted $R^{2}=0.25$; Table 2 ). 
Table 2. Multiple regression models of daily negative and PA predicted by left amygdala persistence following negative images

\begin{tabular}{|c|c|c|c|c|}
\hline Predictor & $b(\mathrm{SE})$ & $\beta$ & $t$ & $\overline{p \text { value }}$ \\
\hline \multicolumn{5}{|l|}{ NA model: } \\
\hline Left amygdala persistence: negative images & $0.23(0.07) * *$ & 0.48 & 3.20 & 0.006 \\
\hline Left amygdala persistence: neutral images & $0.07(0.07)$ & 0.14 & 1.00 & 0.45 \\
\hline Time between fMRI and diaries & $-0.00(0.00)$ & 0.29 & 1.22 & 0.23 \\
\hline Diary days completed & $-0.07(0.03)$ & -0.28 & -1.89 & 0.07 \\
\hline Race & $-0.04(0.09)$ & -0.08 & -0.39 & 0.70 \\
\hline \multicolumn{5}{|l|}{ PA model: } \\
\hline Left amygdala persistence: negative images & $-0.97(0.21) * * *$ & -0.67 & -4.69 & $<0.001$ \\
\hline Left amygdala persistence: neutral images & $-0.08(0.21)$ & -0.06 & -0.40 & 0.73 \\
\hline Left amygdala persistence: positive images & $-0.11(0.20)$ & -0.08 & -0.56 & 0.70 \\
\hline Time between fMRI and diaries & $-0.00(0.00)$ & -0.11 & -0.31 & 0.63 \\
\hline Diary days completed & $0.02(0.10)$ & 0.02 & 0.15 & 0.88 \\
\hline
\end{tabular}

$\mathrm{df}=37, * p<0.05, * * p<0.01, * * * p<.001$. Mean daily NA is square root transformed in this model. Five dummy-coded variables to represent the five sets of twins in the sample were included in the model and not shown here. Race was coded as $1=$ White; $0=$ African American. Gender was coded as $0=$ male; $1=$ female. NA, negative affect; PA, positive affect.

\section{Links between amygdala persistence and PWB}

Pearson bivariate correlations (Table 1) revealed that left amygdala persistence following negative images was not directly associated with PWB $(r=-0.16, p=0.540)$. Similarly, right amygdala persistence following negative images was not associated with PWB $(r=0.05, p=0.810)$. Left amygdala persistence following neutral images was also not associated with PWB $(r=-0.03$, $p=0.874$ ), nor was left amygdala persistence following positive images related to $\mathrm{PWB}(r=-0.14, p=0.600)$. Further, right amygdala persistence following neutral images was not significantly associated with PWB $(r=-0.18, p=0.480)$, nor was right amygdala persistence following positive images related to PWB $(r=-0.03, p=0.868)$.

\section{Links between daily affect and PWB}

Pearson bivariate correlations (Table 1) showed that PWB was significantly associated with daily mean PA $(r=0.51, p<0.001)$, but not mean NA $(r=-0.18, p=0.480)$.

\section{Daily PA links left amygdala persistence following negative images to PWB}

Because there was not a significant direct association between PWB and daily mean NA, we only included daily mean PA in the full path models. In the first model using the full sample, and in line with the bivariate correlations reported above, there was no direct effect of left amygdala persistence following negative images on the first PWB measurement $(b=1.75, z=0.84$, $p=0.517$; Table 3$)$. However, there were significant effects of both left amygdala persistence on mean PA $(b=-0.97, z=$ $-5.49, p<0.001)$, and mean PA on PWB $(b=4.83, z=3.79$, $p<0.001)$. Furthermore, there was a significant indirect effect of left amygdala persistence on PWB through mean PA $(b=-4.68$, $z=-3.12, p=0.005$; Fig. 3). This model accounted for $43 \%$ of the variance in mean $\mathrm{PA}$ and $44 \%$ of the variance in the first measurement of PWB.

We also tested whether the indirect effect was also present using the second PWB measurement approximately seven years later as the outcome in a subset of 31 participants. This model overcomes the temporal limitations of using the first measurement of PWB, which was collected before the fMRI and daily
Table 3. Path model of left amygdala persistence following negative images on the first measure of PWB through daily PA

\begin{tabular}{lrrrccr}
\hline Path & \multicolumn{1}{l}{$b$ (SE) } & \multicolumn{1}{c}{$\beta$} & \multicolumn{1}{c}{ Z } & $95 \%$ lower Cl & $95 \%$ upper Cl & $p$ value \\
\hline a & $-0.97(0.18) * * *$ & -0.67 & -5.49 & -1.32 & -0.62 & $<0.001$ \\
b & $4.83(1.28) * * *$ & 0.52 & 3.79 & 2.33 & 7.32 & $<0.001$ \\
c & $1.75(2.09)$ & 0.13 & 0.84 & -2.34 & 5.84 & 0.517 \\
$c^{\prime}$ & $-4.68(1.50) * *$ & -0.35 & -3.12 & -7.63 & -1.74 & 0.005 \\
Total & $-2.93(1.89)$ & -0.22 & -1.55 & -6.64 & 0.77 & 0.120
\end{tabular}

$N=52$. Path $a$ is the effect of left amygdala persistence on mean daily $P A$, path $b$ is the effect of mean daily PA on PWB, and path $c$ is the effect of left amygdala persistence on PWB. Path $c^{\prime}$ is the indirect effect of left amygdala persistence on PWB through mean daily PA. Covariates included in the model were age, gender, race, twin status, number of diaries completed, and time between assessments; PWB, psychological well-being; PA, positive affect $* p<0.05, * * p<0.01, * * * p<0.001$

diaries. A similar pattern of effects was found when repeating the model. There were significant effects of both left amygdala persistence on mean PA $(b=-0.88, z=-3.29, p=0.003)$, and mean PA on PWB $(b=5.64, z=4.34, p<0.001)$ yielding a significant indirect effect of left amygdala persistence on PWB through mean PA $(b=-4.94, z=-2.62, p=0.016)$. Unlike the previous model, there was a direct effect of left amygdala persistence following negative images on the second PWB measurement $(b=4.72, z=2.19, p=0.047)$. This model accounted for $46 \%$ of the variance in mean $\mathrm{PA}$ and $57 \%$ of the variance in the first measurement of PWB, suggesting that amygdala persistence is indirectly related to future PWB years later through daily PA.

\section{Univariate amygdala difference scores between images and} neutral facial expressions

Overall, we did not find that univariate differences scores between IAPS images and subsequent faces were related to daily affect or PWB. Specifically, the left amygdala difference scores between negative images and the neutral facial expressions that followed were not significantly related to daily NA $(r=0.03$, $p=0.94)$, daily PA $(r=0.03, p=0.94)$, or $\mathrm{PWB}(r=-0.27$, $p=0.20)$. Left amygdala difference scores between the neutral IAPs images and the faces that follow were also not associated with daily NA $(r=0.09, p=0.79)$, daily PA $(r=0.08, p=0.84)$, or PWB $(r=-0.18, p=0.46)$. Further, left amygdala difference scores between the positive IAPs images and the subsequent faces were not significantly related to daily NA $(r=-0.01$, 

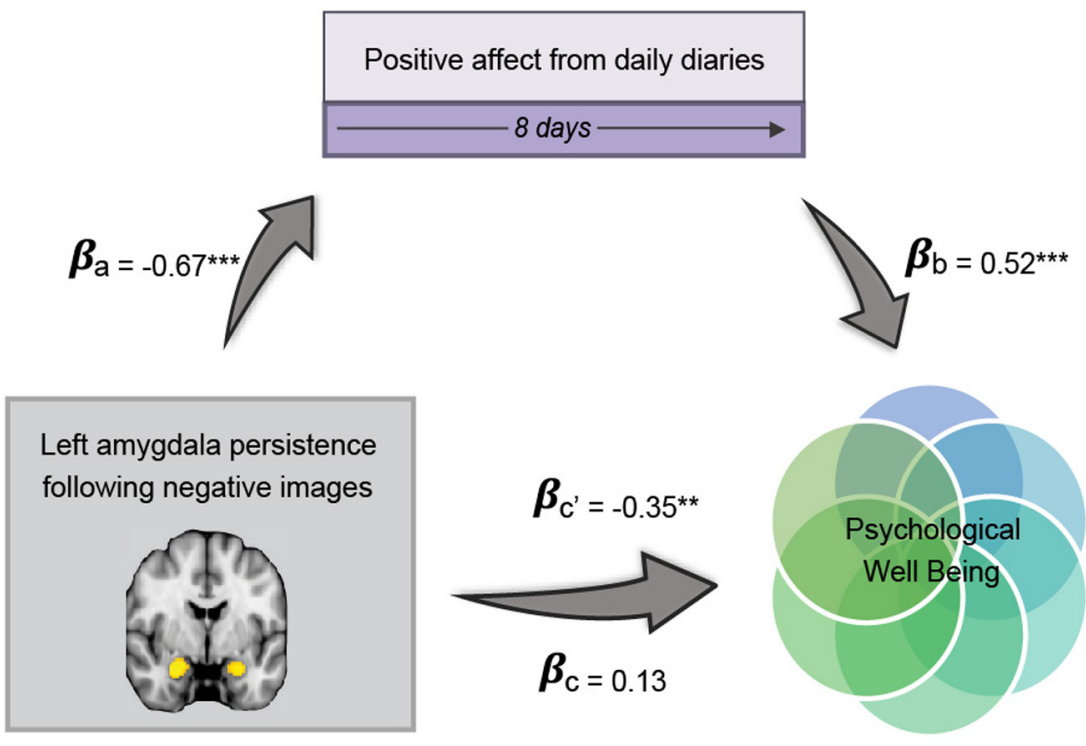

Figure 3. Path model of left amygdala persistence following negative images predicting PWB via daily PA. Standardized path coefficients displayed. Covariates, including age, gender, number of diaries completed, race, twin status, time between assessments and persistence following neutral and positive images are not shown here for simplicity. The indirect path is denoted with $c^{\prime} . N=52$; PWB, psychological well-being; PA, positive affect $* p<0.05$, $* * p<0.01, * * * p<0.001$.

Table 4. Pearson bivariate correlations between PWB, daily affect, and persistence within other brain regions

\begin{tabular}{lccr}
\hline Variable & 1 & 2 & \multicolumn{1}{l}{3} \\
\hline 1. PWB & & & \\
2. Mean daily PA & $0.51 * *$ & & \\
3. Mean daily NA & -0.18 & $-0.66 * *$ & \\
4. L. Occ. persistence: negative images & -0.05 & -0.18 & 0.09 \\
5. L. Occ. persistence: neutral images & 0.20 & 0.07 & -0.23 \\
6. L. Occ. persistence: positive images & 0.29 & 0.11 & -0.07 \\
7. R. Occ. persistence: negative images & 0.12 & -0.11 & 0.07 \\
8. R. Occ. persistence: neutral images & 0.04 & -0.05 & -0.14 \\
9. R. Occ. persistence: positive images & 0.20 & 0.24 & -0.17 \\
10. L. NAcc. persistence: negative images & $-0.57 * *$ & $-0.51 * *$ & 0.27 \\
11. L. NAcc. persistence: neutral images & -0.09 & -0.25 & 0.21 \\
12. L. NAcc. persistence: positive images & 0.02 & 0.09 & 0.04 \\
13. R. NAcc. persistence: negative images & -0.10 & 0.10 & -0.16 \\
14. R. NAcc. persistence: neutral images & -0.20 & -0.05 & -0.17 \\
15. R. NAcc. persistence: positive images & -0.23 & -0.02 & 0.12 \\
16. mPFC persistence: negative images & -0.07 & -0.11 & 0.09 \\
17. mPFC persistence: neutral images & 0.04 & 0.03 & 0.04 \\
18. mPFC persistence: positive images & -0.25 & -0.14 & -0.01 \\
\hline
\end{tabular}

$N=52$. Mean daily NA is square root transformed here. $R$, right; $L$, left; Occ, occipital; NAcc, nucleus accumbens; mPFC, medial prefrontal cortex; NA, negative affect; PA, positive affect $* p<0.05, * * p<0.01$, $* * * p<0.001$.

$p=0.96)$, daily PA $(r=0.20, p=0.42)$, or $\mathrm{PWB}(r=0.12, p=0.66)$. Similarly, univariate difference scores in the right amygdala between negative images and the neutral facial expressions were not associated with daily NA $(r=0.05 p=0.94)$, daily PA $(r=0.02, p=0.96)$, or PWB $(r=-0.16, p=0.52)$. Additionally, right amygdala difference scores between neutral images and the faces that followed were not related to daily NA $(r=0.01$, $p=0.96)$, daily PA $(r=0.04, p=0.94)$, or PWB $(r=-0.22$, $p=0.36)$. Finally, right amygdala difference scores between the positive IAPs images and the subsequent faces were not significantly related to daily NA $(r=-0.05, p=0.94)$, daily PA $(r=0.13, p=0.66)$, or PWB $(r=0.05, p=0.94)$.
Similar to the RSA persistence metric, we tested whether the left amygdala univariate difference scores would predict individual differences in daily affect using multiple regression with relevant covariates. The univariate difference between negative images and neutral facial expressions that followed did not significantly predict daily mean NA $(b=0.04$, $\left.t_{(38)}=0.21, p=0.839, B=0.04\right)$ in a model that also included the univariate difference between neutral images and neutral facial expressions that followed $\left(b=-0.02, t_{(38)}=-0.11, p=0.912, B=\right.$ -0.02 ), the univariate difference between positive images and neutral facial expressions that followed $\left(b=-0.007, t_{(38)}=-0.03, p=0.973, B=\right.$ $-0.005)$, and the same covariates included in the other analyses (age, gender, race, twin status, the time between visits, and the number of diaries completed). A similar lack of effect resulted from an identical model with daily mean PA as the outcome. The univariate difference between negative images and neutral facial expressions that followed did not significantly predict daily mean PA $\left(b=0.40, \quad t_{(38)}=0.63\right.$, $p=0.534, B=0.12)$, nor did the difference between neutral images and neutral facial expressions that followed $\left(b=-0.11, t_{(38)}=\right.$ $-0.18, p=0.861, B=-0.03)$, or the difference between positive images and neutral facial expressions that followed $(b=$ $\left.0.60, t_{(38)}=0.91, p=0.370, B=0.16\right)$. Together, these results suggest that our RSA persistence metric captured unique individual variance related to daily affective experience.

\section{Exploratory analyses addressing spatial specificity}

To assess whether the association between daily affect and amygdala persistence following negative images was unique to the amygdala, we tested whether these effects were present in other regions of the brain. To do this, we extracted the patterns of the left occipital pole (LOcc) and right occipital pole (ROcc), the left NAcc (LNAcc) and right NAcc (RNAcc), and the mPFC, then conducted an identical RSA analysis using these regions. The Pearson bivariate correlations are displayed in Table 4.

Results revealed that persistence in the LOcc and ROcc following negative images were not associated with daily mean NA (ROcc $r=0.07, p=0.837$; LOcc $r=0.09, p=0.810)$, or mean PA (ROcc $r=$ $-0.11, p=0.774$; LOcc $r=-0.18, p=0.667$ ), or PWB (ROcc $r=0.12, p=0.774$; LOcc $r=-0.05, p=0.872$ ). Occipital persistence following neutral images was also not associated with daily mean NA (ROcc $r=-0.14, p=0.765$; LOcc $r=-0.23$, $p=0.495$ ), mean PA (ROcc $r=-0.05, p=0.872$; LOcc $r=0.07, p=0.837$ ), or PWB (ROcc $r=0.04, p=0.873$; LOcc $r=0.20, p=0.650$ ). Also, occipital persistence following positive images was unrelated to daily mean NA (ROcc $r=-0.17, p=0.671$; LOcc $r=-0.07, p=0.837$ ), mean PA (ROcc $r=0.24, p=0.495$; LOcc $r=0.11, p=0.774$ ), or PWB (ROcc $r=0.20, p=0.644$; LOcc $r=0.29, p=0.322$ ).

Similarly, $\mathrm{mPFC}$ persistence following negative images was unrelated to daily NA $(r=0.09, p=0.810)$, daily PA $(r=-0.11$, $p=0.774)$, or PWB $(r=-0.07, p=0.837)$. Also, mPFC persistence following neutral images was not significantly associated with daily NA $(r=0.04, p=0.873)$, daily PA $(r=0.03, p=0.896)$, or PWB $(r=0.04, p=0.873)$ nor was mPFC persistence following positive images related to daily NA $(r=-0.01, p=0.970)$, daily $\mathrm{PA}(r=-0.14, p=0.765)$, or PWB $(r=-0.25, p=0.400)$. 
Results also showed that RNAcc persistence following negative images was not associated with daily NA $(r=-0.16$, $p=0.700)$, daily PA $(r=0.10, p=0.810)$, or PWB $(r=-0.10$, $p=0.782)$. Persistence following neutral images in the RNAcc was similarly not related to daily NA $(r=-0.17, p=0.668)$, daily $\mathrm{PA}(r=-0.05, p=0.873)$, or PWB $(r=-0.20, p=0.644)$. Further, RNAcc persistence following positive images was unrelated to daily NA $(r=0.12, p=0.774)$, daily PA $(r=-0.02$, $p=0.912)$, or PWB $(r=-0.23, p=0.495)$. We found that LNAcc persistence following negative images was significantly correlated with daily PA $(r=-0.51, p<0.001)$ as well as $\mathrm{PWB}(r=-0.57$, $p<0.001)$, but not with daily NA $(r=0.27, p=0.364)$. However, LNAcc persistence following neutral images was not related to any outcomes, including daily NA $(r=0.21, p=0.612)$, daily PA $(r=-0.25, p=0.400)$, and PWB $(r=-0.09, p=0.810)$. LNAcc persistence following positive image was also not significantly associated with daily NA $(r=0.04, p=0.873)$, daily PA $(r=0.09$, $p=0.810)$, and PWB $(r=0.02, p=0.941)$. These correlations suggest that greater persistence (i.e., similarity between representations of negative images and faces that follow after) in the LNAcc is also related to less PA in daily life and even less PWB.

These outcomes indicate that individual differences in persistence following negative images in the amygdala, but not cortical regions, is linked to daily affect. Additionally, the LNAcc, a subcortical structure that can mediate both appetitive and defensive behaviors (Al-Hasani et al., 2015; Berridge, 2019), shows some overlap with the left amygdala in that it is also correlated with daily PA but not NA.

\section{Exploratory analyses to reduce impact of temporal autocorrelation of $\mathrm{fMRI}$}

Because within-run temporal autocorrelation of fMRI could impact metrics of similarity, we recomputed left amygdala persistence by calculating IAPS, face similarity permutations separately for each scan run and then averaged them together. This metric reflects the mean of all possible between-run image-face comparisons computed separately for negative, neutral, and positive image comparisons with the neutral facial expressions presented 3 s later.

Links between left amygdala persistence and daily affect were similar, though slightly weaker for daily NA. Specifically, the relationship between this alternative metric of persistence and daily NA $\left(b=0.56, t_{(36)}=2.00, p=0.053\right)$ was not quite significant. However, this alternative metric of persistence following negative images was associated with daily PA $\left(b=-2.82, t_{(36)}=-3.22, p=0.003\right)$ in identical models from the primary analysis, which included age, gender, race, twin status, the time between visits, and the number of diaries completed as covariates. Also, similar to the primary analyses, this alternative metric of persistence following negative image was not directly associated with PWB $\left(b=10.45, t_{(37)}=1.52, p=0.128\right)$, but the indirect effect of left amygdala persistence on PWB through daily PA was replicated using this alternative metric of persistence $(b=$ $-12.95, z=-2.76, p=0.006$ ).

\section{Discussion}

What gives rise to individual differences in both transient emotional experiences and enduring PWB across months and years? The field of translational neuroscience asserts that real-world, affective outcomes can be predicted by neural activity observed in controlled, experimental settings (Berkman and Falk, 2013; Shackman et al., 2016). In particular, individual differences in neural activity in regions encoding value and salience, like the amygdala, may be critical. Previous research has shown that more persistent amygdala activity in response to aversive stimuli predicts higher levels of traits including neuroticism (Schuyler et al., 2014). We extended this literature by examining how experimentally-obtained individual differences in amygdala persistence, quantified using RSA, map onto naturalistic daily affect and PWB assessed over long periods of time.

Individuals with greater left amygdala persistence following negative images reported more NA and less PA in daily life. This aligns with previous research demonstrating that the amygdala facilitates rapid, negative appraisals of neutral stimuli preceded by an unrelated, aversive stimulus (Lapate et al., 2016; Tambini et al., 2017; Grupe et al., 2018). Additionally, previous work documenting individual differences in amygdala persistence following emotional stimuli (Siegle et al., 2002; Schuyler et al., 2014) suggests that some individuals may be more susceptible to the biasing of neutral stimuli by unrelated, negative stimuli. In the current study, we cannot confirm the neutral facial expressions were perceived as neutral, however, individual differences in neutral-face perception were accounted for by including neutralimage and positive-image persistence as covariates. These results demonstrate that amygdala similarity between negative images and neutral facial expressions that follow such images is linked to subjective emotional experience outside of the laboratory. It may be that for individuals with greater amygdala persistence, negative moments may become amplified or prolonged by imbuing unrelated moments that follow with a negative appraisal. Ultimately, this persistence could result in more NA and less PA, on average, in daily life. This brainbehavior link between left amygdala persistence and daily affect can inform our understanding of more enduring, longterm evaluations of well-being.

PWB was indirectly linked to left amygdala persistence through daily affect. Those who exhibited less amygdala persistence, reported higher daily PA, which in turn, was associated with more positive PWB evaluations. A significant indirect effect between two variables in the absence of a direct effect can occur for a myriad of reasons (MacKinnon et al., 2000; Shrout and Bolger, 2002; Hayes, 2009; Pardo and Román, 2013). One reason is the length of time between variable measurements (James et al., 2006; Pardo and Román, 2013). It is possible that the extended time interval separating amygdala persistence and PWB measurements ( $M=41$ months) limited our ability to detect a significant direct effect. Another possibility is that amygdala persistence contributes to momentary stimulus appraisals, which are proximal to day-to-day affect. In contrast, PWB is determined by an adjoining set of complex integrative processes that govern how one recollects, integrates, and judges the meaning of these daily experiences. These results build on previous links between daily PA and greater PWB (Burns and Ma, 2015) by tying variability in complex, cognitive evaluations about wellbeing to the emotional landscape of daily life and individual differences in brain function. Critically, this indirect effect held regardless of whether we examined the first measurement of PWB in the full sample or the second measurement of PWB seven years after the fMRI collected in a subset of participants. In our study, lower daily NA was not significantly associated with PWB. It is difficult to know how negative moments are integrated into broader evaluations of one's well-being, but one possibility is that less variability in mean NA relative to mean PA in this sample limited our ability to explain individual differences in NA. Another possibility is that the null result between daily NA and PWB may be due a lack of power, as previous research with over 2000 subjects (Rush et al., 2019) found a link between 
daily NA and PWB. Therefore, additional research should probe the directionality and role of daily NA in linking individual differences in brain function with PWB.

Our persistence results were found in the left amygdala, indicating potential differential affective processing between the left and right amygdala. However, hemispheric differences should be interpreted with caution as only the correlation between amygdala persistence and PA (not NA) was significantly stronger in left amygdala. Still, the left amygdala has been reportedly more involved with cognitive processing of emotional information compared with the right amygdala, which facilitates automatic stimulus detection and processing (Gläscher and Adolphs, 2003; Dyck et al., 2011). Thus, left amygdala persistence may reflect more elaborative, cognitive appraisal of the negative stimuli (Phelps et al., 2001). Another possibility is that the left-lateralized amygdala findings may be an artifact of data preprocessing procedures, such as smoothing and motion correction (Murphy et al., 2020). Given the inconsistencies in the current study and the broader literature, additional research is needed to clarify the potential lateralization in amygdala persistence.

These findings also extend the previous literature by using RSA to quantify amygdala persistence in relationship to psychological functioning. We applied RSA, a multivariate technique (Kriegeskorte et al., 2008; Brooks and Freeman, 2018), to capture individual differences in neural persistence, or the discriminability of neural representations of temporally related stimulus classes. In other words, we quantified, for a given person, how much the voxel-wise representation of negative image stimuli resembled the representation of neutral facial expressions that followed negative stimuli in the amygdala. Previous research on amygdala persistence that averages across amygdala voxels ignores differential activation patterns across the spatially heterogenous amygdala structure. Indeed, we found that univariate left amygdala responses to neutral facial expressions following negative images were not associated with daily NA or PA. However, our persistence metric, derived from an RSA comparing voxel-wise patterns of activity in the amygdala between affective stimuli and neutral facial expressions that followed, did predict daily NA and PA. These dynamic comparisons better match real-world experiences than isolating response to a single stimulus, making them prime targets for quantifying amygdala persistence.

These results suggest numerous avenues for future research. Specifically, additional task features could provide a more comprehensive account of neural persistence. The post-IAPS image stimuli used here were limited to neutral facial expressions, however we cannot be certain that subjects perceived these stimuli as neutral. Because the amygdala is involved in facial processing (Todorov et al., 2008), the social nature of these stimuli may have influenced our metric of persistence. It is important to examine whether other types of putatively neutral stimuli influence amygdala persistence and to collect concurrent subjective ratings of these stimuli. Additionally, including greater variation in the time between affective and neutral stimuli (i.e., 3, 5, and 7 s) could pinpoint the optimal lag for detecting individual variation in persistence. Although we used individuals' neutral and positive persistence values as covariates, greater exploration of individual factors that influence amygdala persistence, such as attentional state and hemodynamic response function shape, could deepen our understanding of the mechanisms of neural persistence. Additional work should examine persistence both in the presence and the absence of subsequent, intervening stimuli to better understand possible influences on enduring emotional representations over time.

Beyond the fMRI task design, additional research should further probe the mechanisms by which amygdala persistence translates to daily affect by exploring the cortical-subcortical relationships that explain individual differences in amygdala persistence. Moreover, the amygdala persistence effects here should be replicated with higher spatial resolution scans. In this study, the voxel size was relatively large and sampling amygdala activity with higher spatial resolution would increase the number of voxels included yielding a richer analysis. Beyond the amygdala, other brain regions should be further investigated. Although persistence in cortical regions did not show significant relationships with daily affect and PWB, LNAcc persistence was negatively related to daily PA, similar to the left amygdala. This suggests that persistence-affect relationships may not be specific to the amygdala. Also, while this subset of the MIDUS sample captured a range of adults (ages 39-76), it would be useful to examine these effects during periods of critical development, such as adolescence (Steinberg, 2005). Finally, although daily diary protocols often map on well to momentary experience (Kahneman et al., 2004), ecological momentary assessments across a longer timescale might provide a more fine-grained measurement of daily affective experiences.

In summary, individual differences in amygdala persistence were linked to meaningful, ecological affective outcomes. Specifically, the results indicated that those with less persistence in the left amygdala following negative images may be less susceptible to the biasing of neutral facial expression by unrelated, negative stimuli. Protection against this vulnerability to negative biasing may bring about more PA as well as less NA in daily life. Critically, it is through greater daily PA that left amygdala persistence is related to PWB. As PWB is a complex, cognitive selfevaluation requiring one to integrate across a lifetime of experiences, such an evaluative process is likely supported by distributed brain networks rather than any one individual region.

\section{References}

Al-Hasani R, McCall JG, Shin G, Gomez AM, Schmitz GP, Bernardi JM, Pyo CO, Park SI, Marcinkiewcz CM, Crowley NA, Krashes MJ, Lowell BB, Kash TL, Rogers JA, Bruchas MR (2015) Distinct subpopulations of nucleus accumbens dynorphin neurons drive aversion and reward. Neuron 87:1063-1077.

Avants BB, Tustison NJ, Song G, Cook PA, Klein A, Gee JC (2011) A reproducible evaluation of ANTs similarity metric performance in brain image registration. Neuroimage 54:2033-2044.

Baas D, Aleman A, Kahn RS (2004) Lateralization of amygdala activation: a systematic review of functional neuroimaging studies. Brain Res Brain Res Rev 45:96-103.

Baron RM, Kenny DA (1986) The moderator-mediator variable distinction in social psychological research: conceptual, strategic, and statistical considerations. J Pers Soc Psychol 51:1173-1182.

Benjamini Y, Hochberg Y (1995) Controlling the false discovery rate: a practical and powerful approach to multiple testing. J R Stat Soc Series B Stat Methodol 57:289-300.

Berkman ET, Falk EB (2013) Beyond brain mapping: using neural measures to predict real-world outcomes. Curr Dir Psychol Sci 22:45-50.

Berridge KC (2019) Affective valence in the brain: modules or modes? Nat Rev Neurosci 20:225-234

Brooks JA, Freeman JB (2018) Conceptual knowledge predicts the representational structure of facial emotion perception. Nat Hum Behav 2:581-591.

Burns RA, Ma J (2015) Examining the association between psychological wellbeing with daily and intra-individual variation in subjective wellbeing. Pers Individ Dif 82:34-39.

Charles ST, Mogle J, Urban EJ, Almeida DM (2016) Daily events are important for age differences in mean and duration for negative affect but not positive affect. Psychol Aging 31:661-671. 
Charles ST, Mogle J, Leger KA, Almeida DM (2019) Age and the factor structure of emotional experience in adulthood. J Gerontol B Psychol Sci Soc Sci 74:419-429.

Christensen L, Mendoza JL (1986) A method of assessing change in a single subject: An alteration of the RC index. Behav Ther 17:305-308.

Cox RW (1996) AFNI: software for analysis and visualization of functional magnetic resonance neuroimages. Comput Biomed Res 29:162-173.

Davis M, Whalen PJ (2001) The amygdala: vigilance and emotion. Mol Psychiatry 6:13-34.

Desikan RS, Ségonne F, Fischl B, Quinn BT, Dickerson BC, Blacker D, Buckner RL, Dale AM, Maguire RP, Hyman BT, Albert MS, Killiany RJ (2006) An automated labeling system for subdividing the human cerebral cortex on MRI scans into gyral based regions of interest. Neuroimage 31:968-980

Dyck M, Loughead J, Kellermann T, Boers F, Gur RC, Mathiak K (2011) Cognitive versus automatic mechanisms of mood induction differentially activate left and right amygdala. Neuroimage 54:2503-2513.

Gläscher J, Adolphs R (2003) Processing of the arousal of subliminal and supraliminal emotional stimuli by the human amygdala. J Neurosci 23:10274-10282.

Grupe DW, Schaefer SM, Lapate RC, Schoen AJ, Gresham LK, Mumford JA, Davidson RJ (2018) Behavioral and neural indices of affective coloring for neutral social stimuli. Soc Cogn Affect Neurosci 13:310-320.

Hayes AF (2009) Beyond Baron and Kenny: statistical mediation analysis in the new millennium. Commun Monogr 76:408-420.

Heller AS, van Reekum CM, Schaefer SM, Lapate RC, Radler BT, Ryff CD, Davidson RJ (2013) Sustained striatal activity predicts eudaimonic wellbeing and cortisol output. Psychol Sci 24:2191-2200.

Infantolino ZP, Luking KR, Sauder CL, Curtin JJ, Hajcak G (2018) Robust is not necessarily reliable: from within-subjects fMRI contrasts to betweensubjects comparisons. Neuroimage 173:146-152.

James LR, Mulaik SA, Brett JM (2006) A tale of two methods. Organ Res Methods 9:233-244.

Jenkinson M, Beckmann CF, Behrens TEJ, Woolrich MW, Smith SM (2012) FSL. Neuroimage 62:782-790.

Kahneman D, Krueger AB, Schkade DA, Schwarz N, Stone AA (2004) A survey method for characterizing daily life experience: the day reconstruction method. Science 306:1776-1780.

Kline RB (2015) Principles and practice of structural equation modeling. New York: Guilford Publications.

Kober H, Barrett LF, Joseph J, Bliss-Moreau E, Lindquist K, Wager TD (2008) Functional grouping and cortical-subcortical interactions in emotion: a meta-analysis of neuroimaging studies. Neuroimage 42:998-1031.

Kriegeskorte N, Bandettini P (2007) Analyzing for information, not activation, to exploit high-resolution fMRI. Neuroimage 38:649-662.

Kriegeskorte N, Mur M, Ruff DA, Kiani R, Bodurka J, Esteky H, Tanaka K, Bandettini PA (2008) Matching categorical object representations in inferior temporal cortex of man and monkey. Neuron 60:1126-1141.

Lang PJ, Bradley MM, Cuthbert BN (2008) International affective picture system (IAPS): affective ratings of pictures and instruction manual. Technical Report A-8. Gainesville: University of Florida.

Lapate RC, Rokers B, Tromp DPM, Orfali NS, Oler JA, Doran ST, Adluru N, Alexander AL, Davidson RJ (2016) Awareness of emotional stimuli determines the behavioral consequences of amygdala activation and amygdala-prefrontal connectivity. Sci Rep 6:25826.

Lapate RC, Samaha J, Rokers B, Hamzah H, Postle BR, Davidson RJ (2017) Inhibition of lateral prefrontal cortex produces emotionally biased first impressions: a transcranial magnetic stimulation and electroencephalography study. Psychol Sci 28:942-953.

LeDoux JE (1996) The emotional brain: the mysterious underpinnings of emotional life. New York: Simon and Schuster.

LeDoux JE (2000) Emotion circuits in the brain. Annu Rev Neurosci 23:155-184.

Lindquist MA, Meng Loh J, Atlas LY, Wager TD (2009) Modeling the hemodynamic response function in fMRI: efficiency, bias and mis-modeling. Neuroimage 45:S187-S198.

MacKinnon DP, Krull JL, Lockwood CM (2000) Equivalence of the mediation, confounding and suppression effect. Prev Sci 1:173-181.
Messer K, Matas J, Kittler J, Luettin J, Maitre G (1999) XM2VTSDB: the extended M2VTS database. Available from http://www.ee.surrey.ac.uk/ Research/VSSP/xm2vtsdb.

Murphy JE, Yanes JA, Kirby LAJ, Reid MA, Robinson JL (2020) Left, right, or bilateral amygdala activation? How effects of smoothing and motion correction on ultra-high field, high-resolution functional magnetic resonance imaging (fMRI) data alter inferences. Neurosci Res 150:51-59.

Nili H, Wingfield C, Walther A, Su L, Marslen-Wilson W, Kriegeskorte N (2014) A toolbox for representational similarity analysis. PLoS Comput Biol 10:e1003553.

Ölander K, Muukkonen I, Numminen J, Salmela V (2017) Representational similarity analysis of EEG and fMRI responses to face identities and emotional expressions. J Vis 17:271.

Pardo A, Román M (2013) Reflections on the Baron and Kenny model of statistical mediation. Anales de Psicologia 29:614-623.

Phelps EA, O'Connor KJ, Gatenby JC, Gore JC, Grillon C, Davis M (2001) Activation of the left amygdala to a cognitive representation of fear. Nat Neurosci 4:437-441.

Radler BT, Rigotti A, Ryff CD (2018) Persistently high psychological well-being predicts better HDL cholesterol and triglyceride levels: findings from the midlife in the U.S. (MIDUS) longitudinal study. Lipids Health Dis 17:1.

Raudenbush SW, Rowan B, Kang SJ (1991) A multilevel, multivariate model for studying school climate with estimation via the EM algorithm and application to U. S. high-school data. J Educ Stat 16:295-330.

Revelle W (2020) psych: procedures for personality and psychological research, Northwestern University, Evanston, Illinois, USA. R Package version 1.8.12. Available from https://CRAN.R-project.org/package=psych.

Rosseel Y (2012) lavaan: an R package for structural equation modeling. J Stat Soft 48:1-36.

Rush J, Rast P, Almeida DM, Hofer SM (2019) Modeling long-term changes in daily within-person associations: an application of multilevel SEM. Psychol Aging 34:163-176.

Ryff CD (1989) Happiness is everything, or is it? Explorations on the meaning of psychological well-being. J Pers Soc Psychol 57:1069-1081.

Ryff CD, Keyes CLM (1995) The structure of psychological well-being revisited. J Pers Soc Psychol 69:719-727.

Sander D, Grafman J, Zalla T (2003) The human amygdala: an evolved system for relevance detection. Rev Neurosci 14:303-316.

Satterthwaite TD, Elliott MA, Gerraty RT, Ruparel K, Loughead J, Calkins ME, Eickhoff SB, Hakonarson H, Gur RC, Gur RE, Wolf DH (2013) An improved framework for confound regression and filtering for control of motion artifact in the preprocessing of resting-state functional connectivity data. Neuroimage 64:240-256.

Schuyler BS, Kral TRA, Jacquart J, Burghy CA, Weng HY, Perlman DM, Bachhuber DRW, Rosenkranz MA, MacCoon DG, van Reekum CM, Lutz A, Davidson RJ (2014) Temporal dynamics of emotional responding: amygdala recovery predicts emotional traits. Soc Cogn Affect Neurosci 9:176-181.

Shackman AJ, Tromp DPM, Stockbridge MD, Kaplan CM, Tillman RM, Fox AS (2016) Dispositional negativity: an integrative psychological and neurobiological perspective. Psychol Bull 142:1275-1314.

Shrout PE, Bolger N (2002) Mediation in experimental and nonexperimental studies: new procedures and recommendations. Psychol Methods 7:422-445.

Siegle GJ, Steinhauer SR, Thase ME, Stenger VA, Carter CS (2002) Can't shake that feeling: event-related fMRI assessment of sustained amygdala activity in response to emotional information in depressed individuals. Biol Psychiatry 51:693-707.

Steiger JH (1980) Tests for comparing elements of a correlation matrix. Psychol Bull 87:245-251.

Steinberg L (2005) Cognitive and affective development in adolescence. Trends Cogn Sci 9:69-74.

Tambini A, Rimmele U, Phelps EA, Davachi L (2017) Emotional brain states carry over and enhance future memory formation. Nat Neurosci 20:271-278.

Todorov A, Baron SG, Oosterhof NN (2008) Evaluating face trustworthiness: a model based approach. Soc Cogn Affect Neurosci 3:119-127.

van Reekum CM, Schaefer SM, Lapate RC, Norris CJ, Tun PA, Lachman ME, Ryff CA, Davidson RJ (2018) Aging is associated with a prefrontal lateralmedial shift during picture-induced negative affect. Soc Cogn Affect Neurosci 13:156-163. 